\title{
Erratum to: Constraining theories of gravity by GINGER experiment
}

\author{
Salvatore Capozziello ${ }^{1,6, a}{ }^{10}$, Carlo Altucci ${ }^{1}$, Francesco Bajardi ${ }^{1}$, Andrea Basti ${ }^{2,3}$, \\ Nicolò Beverini $^{2}$, Giorgio Carelli ${ }^{2}$, Donatella Ciampini ${ }^{2}$, Angela D. V. Di Virgilio ${ }^{3}$, \\ Francesco Fuso $^{2}$, Umberto Giacomelli ${ }^{3}$, Enrico Maccioni ${ }^{2,3}$, Paolo Marsili ${ }^{2}$, \\ Antonello Ortolan ${ }^{4}$, Alberto Porzio ${ }^{5}$, Andrea Simonelli ${ }^{3}$, Giuseppe Terreni ${ }^{3}$, \\ Raffaele Velotta ${ }^{1}$ \\ ${ }^{1}$ Dipartimento di Fisica Ettore Pancini, Università di Napoli Federico II and INFN sez. di Napoli, Complesso \\ Univ. Monte Sant'Angelo, via Cintia, Napoli, Italy \\ 2 Dipartimento di Fisica Enrico Fermi, Università di Pisa, Largo B. Pontecorvo 3, Pisa, Italy \\ 3 INFN Sez. di Pisa, Largo B. Pontecorvo 3, Pisa, Italy \\ 4 INFN-National Laboratories of Legnaro, viale dell'Università 2, 35020 Legnaro, PD, Italy \\ ${ }^{5}$ CNR-SPIN and INFN, Napoli, Complesso Univ. Monte Sant'Angelo, via Cintia, Napoli, Italy \\ ${ }^{6}$ Scuola Superiore Meridionale, Largo S. Marcellino 10, 80138 Napoli, Italy
}

(C) The Author(s), under exclusive licence to Società Italiana di Fisica and Springer-Verlag GmbH Germany, part of Springer Nature 2021

Erratum to: Eur. Phys. J. Plus (2021) 136:394

https://doi.org/10.1140/epjp/s13360-021-01373-4

In the original publication of the article most of the authors had been linked to the wrong affiliation.

The original article has been corrected.

The original article can be found online at https://doi.org/10.1140/epjp/s13360-021-01373-4.

a e-mail: capozziello@na.infn.it (corresponding author) 